Wang Y, Gao B, Woo W, Tian GY, Maldague X, Zheng L, Guo Z, Zhu Y. Thermal Pattern Contrast Diagnostic of Micro Cracks with Induction Thermography for Aircraft Braking Components. IEEE Transactions on Industrial Informatics (2018)

DOI link

https://doi.org/10.1109/TII.2018.2802046

ePrints link

http://eprint.ncl.ac.uk/246374

Date deposited

$27 / 02 / 2018$

Copyright

(C) 2018 IEEE. Personal use of this material is permitted. Permission from IEEE must be obtained for all other uses, in any current or future media, including reprinting/republishing this material for advertising or promotional purposes, creating new collective works, for resale or redistribution to servers or lists, or reuse of any copyrighted component of this work in other works. 


\title{
Thermal Pattern Contrast Diagnostic of Micro Cracks with Induction Thermography Thermography for Aircraft Braking Components
}

\author{
Yizhe Wang, Bin Gao (Corresponding Author), Senior Member, IEEE, Wai lok Woo, Senior Member, \\ IEEE, Guiyun Tian, Senior Member, IEEE, Xavier Maldague, Senior Member, IEEE, Li Zheng, Zheyou \\ Guo and Yuyu Zhu
}

\begin{abstract}
Reciprocating impact load leads to plastic deformation on the surface of the kinematic chains in aircraft brake system. As a result, this causes fatigue and various complex natural damages. Due to the complex surface conditions and the coexistence damages, it is extremely difficult to diagnose micro cracks by using conventional thermography inspection methods. In this paper, the Thermal Pattern Contrast (TPC) method is proposed for weak thermal signal detection using eddy current pulsed thermography (ECPT). In this process, the extraction and subsequent separation differentiate a maximum of the thermal spatial-transient pattern between defect and non-defect areas. Specifically, a successive optical flow $(\mathrm{OF})$ is established to conduct a projection of the thermal diffusion. This directly gains the benefits of capturing the thermal propagation characteristics. It enables us to build the motion context connected between the local and the global thermal spatial pattern. Principal Component Analysis (PCA) is constructed to further mine the spatial-transient patterns to enhance the detectability and sensitivity in micro crack detection. Finally, experimental studies have been conducted on an artificial crack in a steel sample and on natural fatigue cracks in aircraft brake components in order to validate the proposed method.
\end{abstract}

Index Terms-Aircraft braking system, Induction thermography, Optical flow, Principal component analysis, Thermal pattern contrast, Weak signal extraction

\section{INTRODUCTION}

In the last few decades, infrared thermography [1-3] has received vast and growing attention for diagnostics and monitoring in industry. Along with the development of thermal science, the industry has put forward higher requirements, such as in-service inspection, quantification and online measurement with robots [4]. In-service kinematic chains frequently coexist with various types of complex natural defects due to harsh working environments, such as high temperature, stress, and reciprocating impact loads [5]. Due to the complex surface conditions and the coexistence damages, it is extremely difficult to diagnose natural cracks by using conventional thermography inspection method.

The braking system is a functionally independent subsystem of an aircraft. During the braking process, the hydraulic oil from the brake system flows into the cylinder block and then pushes the piston. In addition, it compresses the movable and static pieces alternately arranged in the hub $[6,7]$. The driven key is a significant component in the braking system that connects an airplane wheel to the rotating discs in synchronous rotation [7]. The rotating discs brake the aviation wheels mediated by the driven keys due to the inertia of the airplane in the braking process [8]. This process leads to reciprocating impact from the slot of rotating discs on both sides of the driven keys. The repeated impact damage is part of the fatigue that manifests as a macroscopic plastic deformation. The common failure modes of repeated impact in metals include fatigue cracks [9], particle fragmentation/wear, upsetting, surface corrosion pitting and peeling. Micro cracks are considered intolerable defects and common failure mechanisms [10], which require an early diagnosis and warning for safety and prevention purposes. Therefore, it is crucial to detect and evaluate the fatigue cracks in driven keys in order to guarantee the reliability and integrity of jet braking systems.

Advanced signal processing has been used to improve the detection accuracy and precision in the thermography Nondestructive testing (NDT) [11-16]. This can be categorized in different groups according to the manner in which data is handled [17]. Thermal contrast-based techniques are based on the thermal mechanism. Thermographic Signal Reconstruction (TSR) is a sophisticated thermal contrast-based method which was proposed by Steven M. Shepard in 2001[18]. It improves the spatial and temporal resolution of a thermogram sequence. It enhances the flaw contrast and improves noise rejection qualities. The image enhancement has been conducted by subjecting the raw data upon a set of logarithmic polynomial fitting of the temperature time-evolution. Matrix factorization techniques have been greatly expanded in thermography processing. Principal Component Analysis (PCA) has been successfully used to capture the transient-spatial thermal pattern by considering the initial sequence as either a set of images or a set of temporal profiles [19]. Signal transform methods convert the detection signal into the other domain to seek effective detection information and patterns. Fourier transform was applied to pulsed thermography. It significantly enhances the flaw-contrast by using the phase map and image normalization [20]. Influence of non-uniform heating and surface emissivity variation is removed by using a Fourier transformation based image reconstruction algorithm [21]. The success of these signal processing methods has one common ingredient that bridges the gap between the physical world and mathematical modeling world. For crack detection in the thermal field, several outstanding results based on advanced signal processing have been reported [22-25]. Vrana et. al. researched the mechanisms and models for crack detection with induction thermography [22]. Dudzik proposed a neural algorithm to estimate defect depth using an active thermography [23]. Peng et. al. discussed different angled defects of rolling contact fatigue (RCF) in rails [24]. Gao et. al. focus on the quantitative assessment and error analysis for the surface cracks [25]. However, previous research rarely discusses the diagnosis of natural cracks which are concealed in kinematic chains with complex geometries. It remains a challenge to extract and distinguish the weak thermal features of natural cracks from the samples with poor surface conditions and noise interference.

Eddy Current Pulsed Thermography (ECPT) is a multi-physics coupling NDT method which belongs to the Induction Thermography family. The main physical process of ECPT involves induced eddy currents heating and thermal diffusion [26]. The combination of these two physical fields is beneficial for detecting turbulence in conductive materials by analyzing the thermal patterns. The rich transient information in the ECPT spatial-transient pattern has attracted a wide range of interest [27].

In this paper, a method termed as the Thermal Pattern Contrast (TPC) is proposed to extract and separate the thermal spatial-transient patterns to enhance resolution in order to better detect micro fatigue cracks through induction thermography. TPC is based on a deep understanding of the thermal mechanism. It enables us to build the motion context connected between the local and the global thermal 
spatial patterns which obtains the benefits from the motion extraction context in order to characterize the thermal propagation. It provides a possibility to extract and separate the thermal spatial-transient patterns between defect and non-defect areas for weak thermal signal detection. The drawback of the TPC method is the difficulty to reflect in the depth quantification of a defect. The contributions of this paper can be summarized as follows: 1) building the relationship between inductive heating propagation and thermal-optical flow; 2) modeling and mining of mathematical spatial-transient thermal patterns for automated crack detection. The experiments have been designed to validate the efficacy and robustness of the proposed method. First, an abstract model is built on a steel sample containing artificial cracks. Second, simulating the thermal behavior of micro cracks, which involves weak thermal signal under strong background interference. Finally, the robustness of the proposed method is demonstrated by using eight different sets of natural fatigue cracks to introduce the reciprocating impact loads of the driven keys in aircraft brake systems for quantitative defect detection.

The rest of this paper has been organized as follows: the theory of Thermal Pattern Contrast is described in Section 2. The experiments and results are analyzed and compared in Section 3. Finally, conclusions and further work are outlined in Section 4.

\section{METHODLOGY OF INSPECTION SYSTEM AND IMAGE ANALYSIS}

This section serves as a basic exposition of TPC theory. Firstly, an introduction of ECPT is presented. Secondly, the relationship between thermal diffusion and optical flow is derived. Finally, data reconstruction and the principle of fixed frame-interval is presented for thermal spatial-transient pattern extraction and separation.

\section{A. Eddy current pulsed thermography (ECPT)}

Fig. 1 shows the schematic diagram of ECPT. The excitation signal generated by the induction heating element is a high frequency current pulse continuing during a few milliseconds. It is driven to the transmitter coil above the conductor which will induce the eddy currents and generate the resistive heat in the conductive material. Thermal diffusion leads the heat flow from a high to low temperature area, and then reduces the contrast so as to reach a heat balance in the material. If a defect exists in the conductive material, the distribution of eddy current or the process of thermal diffusion will be disturbed. Consequently, the resultant surface heat distribution and the transient temperature time spatial response will show the variation captured by an infrared camera.

The main physical process of ECPT consists of induced eddy currents heating and thermal diffusion. Electromagnetic governing equation can be transformed by the differential of the Maxwell equations and constitutive equations in electromagnetism [26], namely:

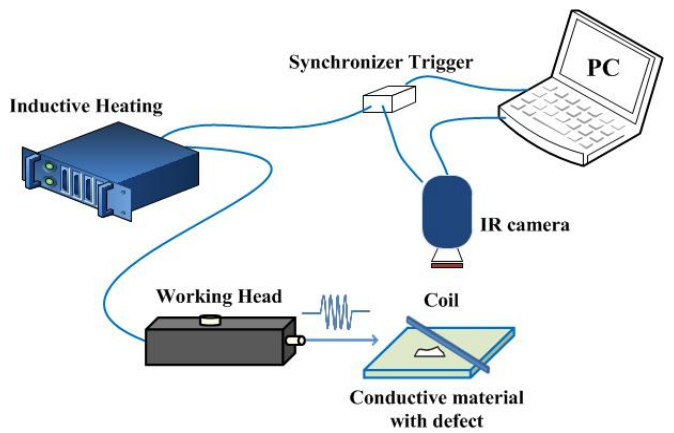

Fig. 1. ECPT schematic diagram

$$
\frac{1}{\mu} \nabla^{2} \mathbf{A}+\sigma \frac{\partial \mathbf{A}}{\partial t}=\mathbf{J}_{s}
$$

After setting the boundary conditions, the distribution of eddy currents induced in the conductor can be solved by using the finite element method (FEM) through (1).

The temperature of a conducting material will increase owing to resistive heating from the induced electric current. This is known as Joule heating which is the coupling of the electromagnetic and thermal fields. The following equation expresses this relationship:

$$
\mathcal{Q}=\frac{1}{\sigma}\left|J_{e}\right|^{2}=\frac{1}{\sigma}|\sigma E|^{2}
$$

The above equation expresses the relationship between the sum of the generated heat $Q$. Due to the energy conservation and Fourier heat conduction, the inductive heat conduction equation can be expressed as:

$$
\frac{\partial T}{\partial t}=\underbrace{\frac{\lambda}{\rho C_{p}}\left(\frac{\partial^{2} T}{\partial x^{2}}+\frac{\partial^{2} T}{\partial y^{2}}+\frac{\partial^{2} T}{\partial z^{2}}\right)}_{\text {Thermal diffusion }}+\underbrace{\frac{1}{\rho C_{p}} q(x, y, z, t)}_{\text {Joule heating }}
$$

where $T=T(x, y, z, t)$ is the transient surface temperature distribution, $\lambda$ is the thermal conductivity of the material (W/m K), $\rho$ is the density $\left(\mathrm{kg} / \mathrm{m}^{3}\right), C_{p}$ is the specific heat capacity $(\mathrm{J} / \mathrm{kg} \mathrm{K})$, and $q(x, y, z, t)$ is the heat generation function per unit volume and unit time, which is the result of the Joule heating by the eddy current. The heat conduction equation is merged with Joule heating and thermal diffusion.

\section{B. Thermal transient pattern}

In the thermal field, optical flow indicates the projection of $3 \mathrm{D}$ heat propagation onto the 2D thermal image. This is shown in Fig. 2. Each pixel $(x, y)$ owns one velocity vector $\vec{w}=(u, v)$ to reveal the speed of thermal diffusion at this pixel which is moving across in a certain direction $(x+u, y+v)$. The mathematical derivation between optical flow and thermal diffusion and the physical mechanism of this bonding will be introduced and explained in the following section.

In this case, the detection signal in the form of the temperature $(T)$ is captured by the infrared camera. The thermal image sequence is constructed as a three-dimension spatial-transient tensor with respect to the location $x$ and $y$, and time $t$. The intensity of each pixel $(x, y)$ is defined as $T(x, y, t)$.

Based on the bright constancy assumption, the Optical Flow Constraint equation is given by:

$$
\frac{\partial T}{\partial x} u+\frac{\partial T}{\partial y} v+\frac{\partial T}{\partial t}=0
$$

The optical flow vector is given by $\vec{w}=(u, v)$, in which $u$ and $v$ are row vectors. The temperature gradient $\nabla T$ is expressed as $\nabla T=\left(T_{\mathrm{x}}, T_{y}\right)=\left(\frac{\partial T}{\partial x}, \frac{\partial T}{\partial y}\right)$. The time derivative $T_{\mathrm{t}}=\frac{\partial T}{\partial t}$. Thus, Equation (11) can be written as:

$$
\nabla T \cdot \mathrm{W}+T_{t}=0
$$

The linearized brightness constancy constraint provides only one equation to recover the two unknown components of $w$. This is known as the aperture problem of the optical flow algorithms. Thus, the Horn-Schunck method [28] is used for the implementation, where the relationship between the optical flow $w$ and the temperature gradient $\nabla T$ is formulated as a global energy function which is solved through minimization.

The above formula establishes the bridge between optical flow and the temperature gradient. According to the Fourier's law of heat conduction [26]:

$$
Q=-\mathrm{k} \cdot \nabla T
$$


The thermal diffusion through a material is negatively proportional to the temperature gradient. Therefore, the optical flow vector displays thermal diffusion, whose direction is opposite to that of the temperature gradient, according to (5) and (6).

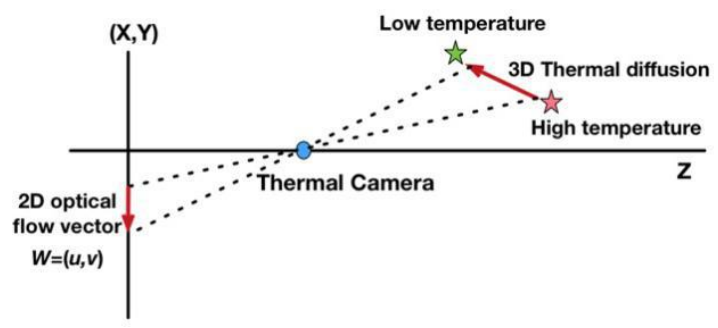

Fig. 2. Relationship between thermal diffusion and optical flow

\section{Feature extraction and separation}

The optical flow characterizes the thermal behavior, however, the result of the optical flow solely expresses the thermal diffusion between two images which only consider spatial information of the thermal diffusion in one moment. Thus, information is lost on the development of the whole thermal transient progress. This leads to the next logical move: exploring sufficient information from the multi domain (spatial-transient) for feature extraction.

The result of optical flow between two frames could be characterized as a two-dimensional array in which each pixel contains vector information: the direction and length of the arrows, representing the heat propagation indirectly. For data reconstitution, every two images from the original thermal image sequence $(Y)$ with a fixed frame-interval in turn calculate the successive optical flow which will generate a new optical flow sequence ( $Y_{O F}$ ) as the input of PCA. This successive optical flow image sequence can be regarded as a three dimensional matrix. The PCA algorithm can be used for defect separation [29]. This mathematical model [27] can be described as:

$$
Y_{O F}(\mathrm{t})=\sum_{i=1}^{N_{s}} m_{i} X_{i}(t)
$$

where $X_{i}(t)$ denotes the characteristic thermal patterns generated by the area represented by position $i$ at time $t$ with dimension $N_{x}$ by $N_{y}$.

An important part of data reconstruction is to select the appropriate fixed frame-interval. The suitable number of fixed frame-intervals $(N)$ depends on the correlation coefficients [30] between defect and non-defect areas. The smaller the value of the correlation coefficients, the better the separation result of PCA.

Fig.3. a) illustrates the flow chart of the entire data processing operation, which contains 1) the preprocessing (noise reduction by substracting of the first frame), 2) selection of the appropriate fixed frame-interval by calculating the correlation coefficient and processing the optical flow, and 3) PCA processing. Fig.3. b) shows the specific selection process of the fixed frame-interval. The minIndex refers to the number of the most suitable interval.

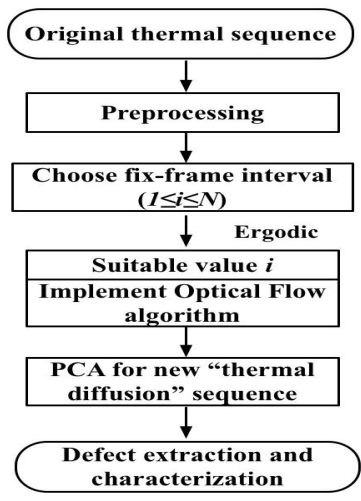

(a)

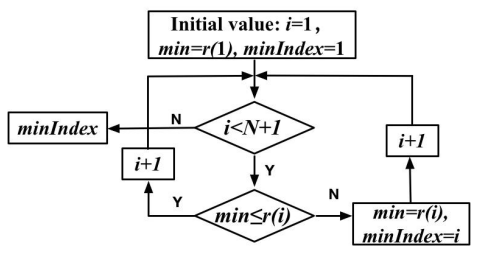

(b)
Fig. 3. a) Flow chart of the data processing of TPC; b) Specific selection process of the optimum fixed frame-interval

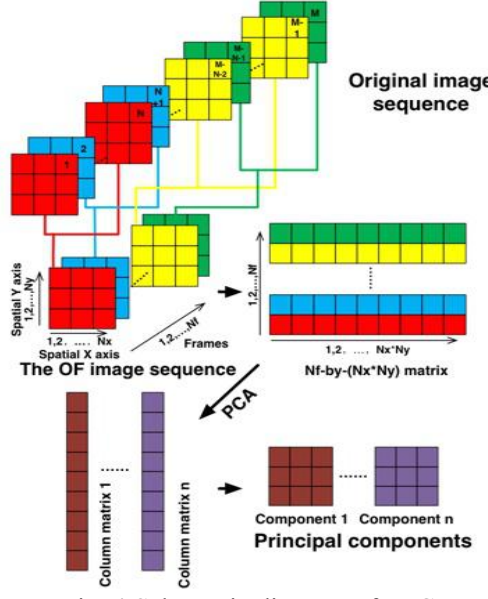

Fig. 4 Schematic diagram of TPC

Fig. 4 shows a schematic diagram of the detailed process of TPC. The input of PCA is generated by new image sequences after the processing of the optical flow between each two images with fixed frame intervals. The number of principal components of PCA is set as $N_{\mathrm{s}}$. Finally, principal components are reconstructed as the spatial-transient patterns for crack detection.

\section{Abstract model}

An abstract model is used to simulate a defect signal which behaves as a weak part and suffers from strong interference. This model enables a better interpretation of the TPC process. Fig. 5 demonstrates the schematic diagram of the abstract model. The induction coil is located at a certain distance $(m)$ from the crack which is perpendicular to the coil. The design purpose of the position between the coil and defects is to generate a weak defect signal in order to imitate the impact damage. This abstract model serves to illustrate the artificial crack sample as discussed in Fig. 6.

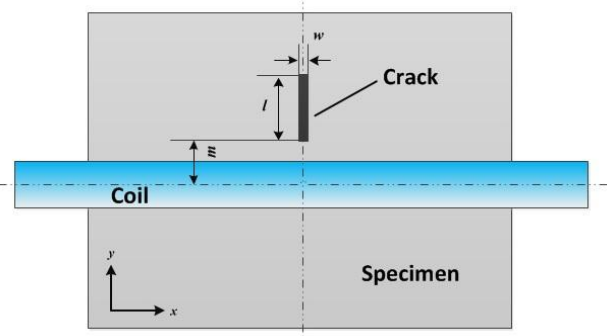

Fig. 5. Top view of the abstract model

\section{RESULTS AND DISCUSSIONS}

\section{A. Experiment set-up}

The Easyheat 224 from Cheltenham Induction Heating, Ltd was used for induction heating, with a maximum excitation power of 2.4 $\mathrm{kW}$, a maximum current of $400 \mathrm{~A}$ and an excitation frequency range of $150-400 \mathrm{kHz}$. The excitation coil was made of $6.35 \mathrm{~mm}$ high-conductivity hollow copper tubing. The infrared system FLIR-A655sc was used to record the temperature change. It is a Stirling uncooled camera with a full frame $640 \times 480$ resolution of 7.5-14 $\mu \mathrm{m} \mathrm{InSb}$ detectors. This camera has a sensitivity of $<50 \mathrm{mK}$ and a maximum full frame rate of $200 \mathrm{~Hz}$. The temperature range is from $-40^{\circ} \mathrm{C}$ to $650^{\circ} \mathrm{C}$.

In the following subsections, two case studies involving a perpendicular crack and fatigue natural cracks under impact damage are provided. In these experiments, the excitation frequency and 
current were set as $256 \mathrm{kHz}$ and $380 \mathrm{~A}$, respectively. The heating time was set as $0.2 \mathrm{~s}$ and the entire recording time was $4 \mathrm{~s}$. Because the emissivity of the sample is unknown, a digital level (dl) is used to describe the temperature rather than Celsius degrees $\left({ }^{\circ} \mathrm{C}\right)$. Specifically, the radiation of the object was sampled by using the commercial thermography software Altair in which the unit of radiation is digital level (DL). A nonlinear transfer function after calibration can convert the radiation (unit: $\mathrm{DL}$ ) into temperature (unit: $\mathrm{K} /{ }^{\circ} \mathrm{C}$ ), which requires an operator setting several parameters such as emissivity background temperature, and transmission. In order to simplify the process, we used DL as a representation of temperature in the experiment.

\section{B. Abstract model-artificial crack in steel sample}

The abstract model serves to simulate the valuable diagnosis information for the detection of a weak thermal signal. Fig.6.(a) demonstrates the abstract model, a steel sample with one perpendicular crack $(l: 10 \mathrm{~mm} w: 0.3 \mathrm{~mm} d: 1 \mathrm{~mm})$ constructed in the form of a rectangular block $(l \times w \times d: 120 \mathrm{~mm} \times 60 \mathrm{~mm} \times 5 \mathrm{~mm})$. During the experiment, both the coil and IR camera were placed above the defect side. The lift-off distance between the coil and sample was $1 \mathrm{~mm}$. The distance $(m)$ between the artificial crack and the excitation coil was equal to $3.5 \mathrm{~mm}$.
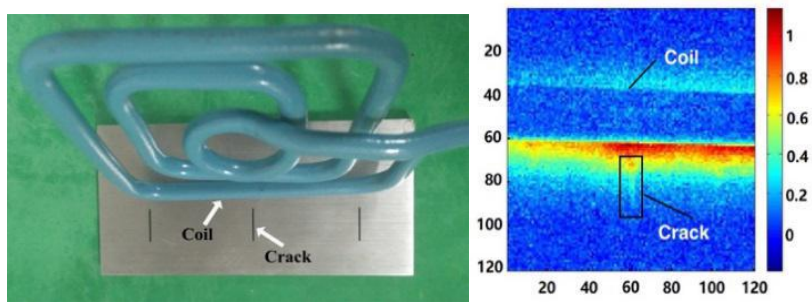

Fig. 6. (a) Artificial crack with perpendicular crack under test; (b) Original transient temperature distribution at $5 \mathrm{~ms}$

Fig.6. (b) presents the results of the transient temperature distribution on the surface of the material at an early heating stage ( 5 $\mathrm{ms}$ ) without any data processing. It shows that the area underneath the excitation coil has a higher temperature than the defect area. The reason is that the distribution of eddy current shows an exponential decay trend from the center of the straight wire to both flanks. The coil and artificial crack are positioned perpendicularly. This results in the hot spot appearing in the tip of the defect due to the diversion of eddy current when it encounters a discontinuity. Concurrently, it is revealed that the lateral thermal diffusion is seen to be feeble not only because of the great distance between the crack and coil but also due to the parallel direction between the thermal diffusion and the defect. Even though the tip of the crack displays a weak hot spot, these thermal features are difficult to recognize due to the strong interference from the high temperature region under the coil.

The result of optical flow is shown in Fig. 7. The defect and non-defect areas present totally different thermal patterns. The arrows of optical flow converge around the defect, which indicate the disturbance of thermal behavior, hindered by the crack. In contrast, the direction of optical flow under the coil emerges as a horizontal translation, which is along the radial orientation of the coil and flows along with the temperature gradient. This result shows that the optical flow has the ability to extract the singular transient thermal behavior which enhances the detection of defect thermal patterns.

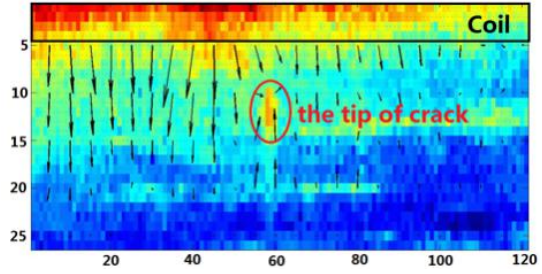

Fig. 7. Result of the optical flow algorithm for the artificial crack
The correlation coefficients [30] are selected as the evaluation criteria to discover the adaptive fixed frame-interval to generate the new optical flow sequence. Unrelated variables will be divided into different components of PCA. The Fig. 8 shows the correlation coefficient between the coil, the crack and the sound area (means non-defect area). The traversal range of the frame interval is from 1 to 80. With the increase of the frame interval, the cross correlation coefficient of coil-coil and crack-crack remained at a high value.

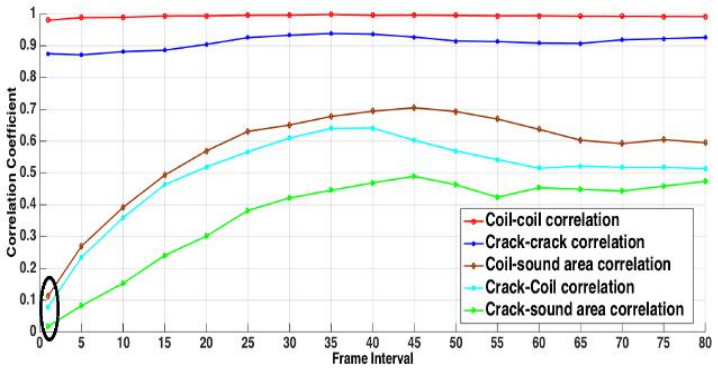

Fig. 8. Result of correlation coefficients for artificial crack

This means that any region of the defect or coil could represent itself. Notwithstanding above, the correlation coefficient between the coil, the crack and the sound area presents an increasing trend with the rise of frame intervals. Thus, the small frame interval has the potential to fully separate the thermal informative patterns as marked in a black circle of Fig. 8. According to the results of the correlation coefficients, one fixed-frame interval is chosen for data reconstruction. Fig. 9 shows the PCA results where the number of principal components is set to eight. This number was given by a Monte-Carlo repeated experiment [31] involving 12 independent trials. The components one and two contain the coil information and the component three represents the tip information of the crack.

These results show that the TPC method has the potential to diagnose the micro crack under strong interference.
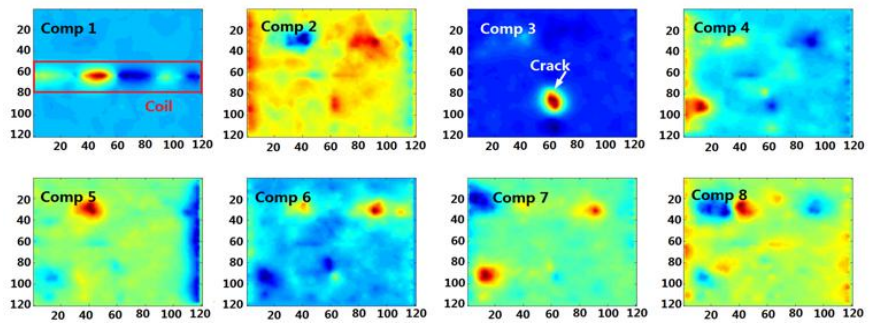

Fig. 9. Result of PCA after Optical Flow under steel sample testing

\section{Natural fatigue cracks in aircraft brake systems}

A driven key, which has been removed from an aircraft in service with multi-cracks on the surface of the impact areas, is shown in Fig. 10. (c). The impact areas with flaws are marked by red rectangles. As illustrated in Fig. 10. (d), the driven key is fixed to bench vice and a pancake coil is used.

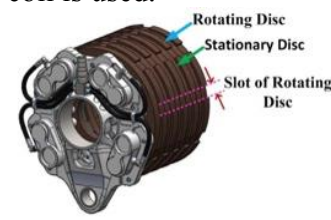

(a)

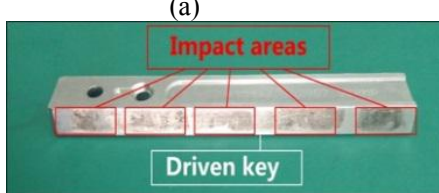

(c)

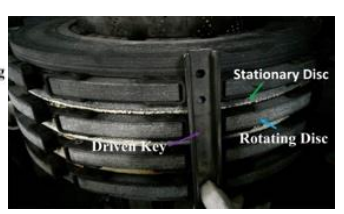

(b)

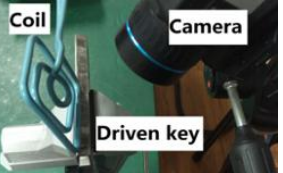

(d)
Fig. 10. (a) Schematic diagram of the braking system;(b) Real structure of the braking system;(c) Driven key sample with impact damage;(d) ECPT experiment setup 
After the preprocessing, the one result of the optical flow (OF) algorithm between two thermal images is shown in Fig. 11. It presents the different thermal patterns between cracks and the sound area. The two white circle regions representing the thermal perturbation are defined as the suspected flaw areas (crack 1 and crack 2). The arrows of optical flow gather together around the defects, which indicate eddy current passing around the cracks and the disturbance of thermal behavior. In contrast, the direction of optical flow under the coil and the sound area without defect emerge as a horizontal translation. Fig.12 shows the results of correlation coefficients for the natural crack detection. The regions of crack 1 and crack 2 are defined in Fig. 13. The results show that crack 1 and crack 2 maintain a high value of correlation coefficients regardless of the variation of the frame interval. Unfortunately, the correlation coefficients between the cracks and coil are also high. The reason is that the thermal pattern of the cracks is affected by the adjacent coil. To separate the thermal behavior of the crack and coil, a relatively small value of correlation coefficients between the cracks and coil should be chosen, so that the difference between the coil and the cracks could be as large as possible. As for the sound area, the value of correlation coefficients should be as small as possible. Therefore, the frame interval was chosen as the range [65, 80], which satisfies the above two conditions so that the value of correlation coefficients is at a minimum between the crack and coil \& sound area. As an example, the 80 fixed-frame interval is demonstrated. The number of principal components of PCA is set to eight. In Fig. 13, the eighth principal component shows that the two fatigue cracks have been separated with minimal interference, and have been marked with red circles.

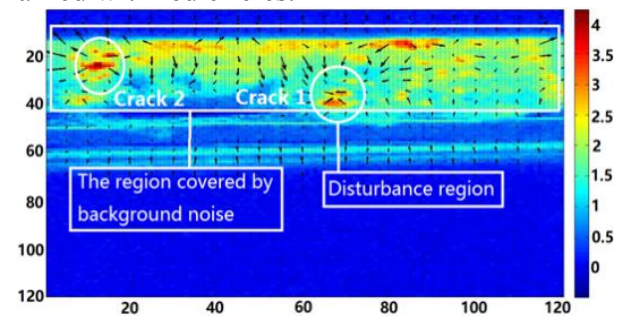

Fig. 11. Result of the optical flow algorithm for the natural crack

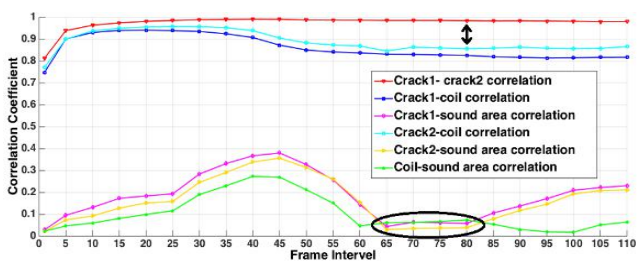

Fig. 12. Result of correlation coefficients for the natural crack
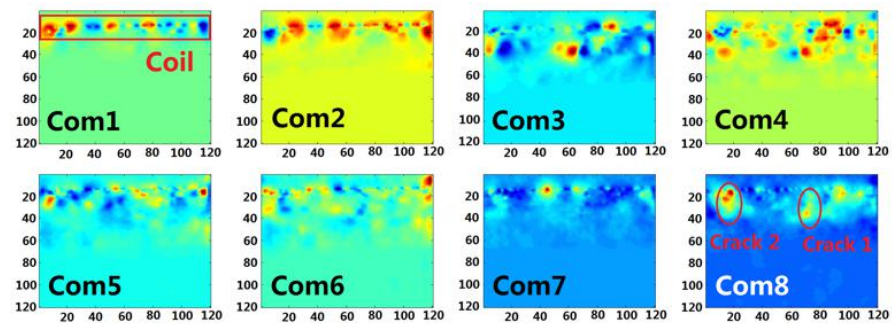

Fig. 13. Result of PCA after Optical Flow under impact damage

To validate the results, magnetic particle testing (MPT) is employed to serve as a validation NDT tool for the detection. Fig.14. (a) illustrates the result of Magnetic Particle Testing. It validates that the flaws exist at the locations with fluorescence as well as two areas circled in Fig.14(b). The comparison diagram shows that the results of the two methods coincide with each other. This result proves that TPC works equally well for natural cracks.

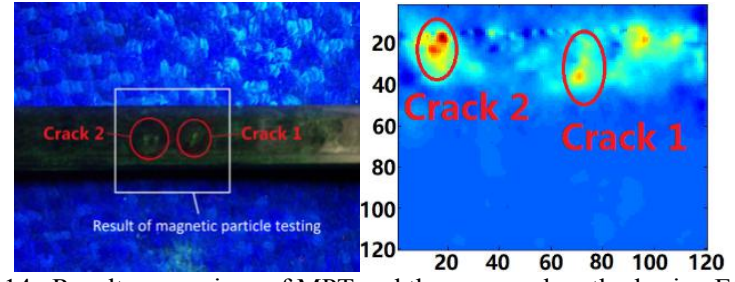

Fig. 14. Result comparison of MPT and the proposed method using ECPT: (a) Magnetic Particle Testing; (b) TPC

To verify the robustness of the proposed method, 8 groups of different specimens have been validated. A detailed description of the 8 groups composed of different specimens is given. All specimens are from the in-service aircraft braking system. All of them contain micro cracks in the impact area. The material properties and structure of the eight specimens are the same. The Magnetic Particle Testing is employed as a standard NDT tool to generate the ground truth of the defects. The position and shape of all cracks in the specimens have been identified using magnetic particle testing (MPT).

The comparison results between different detection approaches are shown in Table 2. These methods include: manual selection of original thermal imaging, TPC, PCA [27], Thermographic Signal Reconstruction (TSR) [21], Fourier analysis (FFT) [32]. In this case, the position of the coil and crack is known as prior information. Based on the results of Table 2, a quantitative analysis is then provided by the events based F-score [33] and SNR [34] using the majority vote method [35].

The events based F-score [33] is defined as follows:

$$
F=\frac{\left(\alpha^{2}+1\right) \times \text { Precision } \times \text { Recall }}{\left(\alpha^{2} \times \text { Precision }\right)+\text { Recall }}
$$

The Precision and Recall is described in Table 1. The $\alpha$ in (8) is a default value that determines the weight of the Precision and Recall. In this paper, the value of $\alpha$ is set to one which means that the Recall is as important as the Precision. In Fig. 15, all of the results of the Fig. 16 will be divided into $6 \times 6$ square grids. According to the results of magnetic particle testing, each region as an event could be determined by the attribute (defect or non-defect) for the calculation of the F-score.

The signal-to-noise ratio (SNR) [34] then further evaluates the thermal contrast between the defective and non-defective region. The calculation of $\operatorname{SNR}(\mathrm{dB})$ is defined in the equation below:

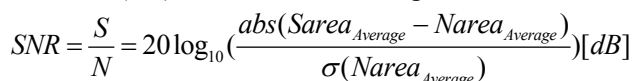

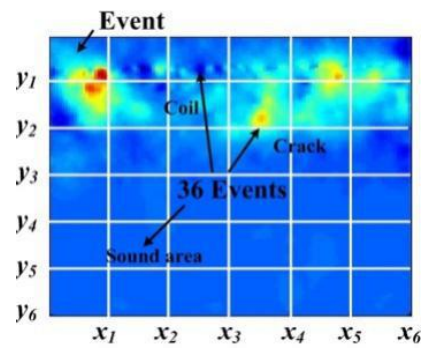

Fig. 15. The grid of the event segmentation for the F-score where Sarea $_{\text {Average }}$ and Narea $a_{\text {Average }}$ are the average temperature in the defect and non-defect regions (the entire image except the defect area), respectively. The $\sigma\left(\right.$ Narea $\left._{\text {Average }}\right)$ is the temperature standard deviation in the non-defective region.

Table 1 The definition of Precision and Recall

True positive (TP)

A defect exists and is detected 


\begin{tabular}{|c|c|}
\hline $\begin{array}{c}\text { False positive (FP) } \\
\text { No defect exists but one is identified }\end{array}$ & Precision $=\frac{T P}{T P+F P}$ \\
\hline $\begin{array}{c}\text { False negative (FN) } \\
\text { A defect exists but is not detected }\end{array}$ & Recall $=\frac{T P}{T P+F N}$ \\
\hline $\begin{array}{c}\text { True negative (TN) } \\
\text { No defect exists and none is detected }\end{array}$ & \\
\hline
\end{tabular}

The quantitative analysis involving the event based F-score and SNR is presented in Table 2. The " $\boldsymbol{x}$ " in SNR results refers to an occurrence in which the method could not detect the micro crack in the corresponding experiment. In the tests 1 and 2, the F-score and SNR of TPC are equal to $85.7 \%$ and $80.0 \%$, with SNR of $12.1 \mathrm{~dB}$ and $12.5 \mathrm{~dB}$, respectively. Compared with FFT, the F-score improves by $35.7 \%$ and $13.3 \%$ and the SNR is enhanced with $3.6 \mathrm{~dB}$ and $13.2 \mathrm{~dB}$, respectively. Nevertheless, the methods of PCA and TSR do not perform a good detection of the micro impact crack. From test 3-5, the results obtained using TPC have obvious superiority. The F-score of TPC is equivalent to $100.0 \%$ which indicates that the micro impact crack is successfully detected. In contrast, other methods have an unsatisfactory performance which still contains the complex background as well as noise information. Meanwhile, compared with the SNR of the remaining methods, the SNR of TPC leads to an improvement of 3.2 $\mathrm{dB}, 9.9 \mathrm{~dB}$ and $7.5 \mathrm{~dB}$ for PCA, $11.7 \mathrm{~dB}$ and 15.7 for TSR, and finally, $10.7 \mathrm{~dB}$ and $10.0 \mathrm{~dB}$ for FFT. In test 6 , all of the above methods basically detected the defect and four signal processing methods have a similar quality behavior. In test 7 and 8 , the PCA process could not distinguish the crack location. The F-score and SNR of TPC is equivalent to $80.0 \%, 100.0 \%$ and $17.3 \mathrm{~dB}, 16.5 \mathrm{~dB}$. Compared with TSR and FFT, the F-score of TPC improves $40 \%$ and $66.7 \%$, respectively. The SNR of TPC is enhanced by $4.1 \mathrm{~dB}$ and $0.5 \mathrm{~dB}$ for TSR, $8.3 \mathrm{~dB}$ and $3.1 \mathrm{~dB}$ for FFT, respectively.
From the overall analysis, the average of the F-score and SNR of TPC is $93.2 \%$ and $15.6 \mathrm{~dB}$. Compared with PCA, TSR and FFT, the average of the TPC F-score improves $59.9 \%, 67.67 \%$ and $45.7 \%$ and SNR improves by $10.7 \mathrm{~dB}, 11.6 \mathrm{~dB}$ and $6.7 \mathrm{~dB}$, respectively. In general, compared to these classical algorithms, the TPC method enhances the extraction of the weak singular thermal spatial-transient pattern and compensates effectively to strengthen the detectability of micro cracks.

\section{CONCLUSION}

This paper proposes a method termed as the Thermal Pattern Contrast to extract and separate thermal spatial-transient patterns in order to diagnose micro cracks. The data processed from the proposed method has been elaborated and analyzed. The data reconstruction has been presented by the successive optical flow between every two images with a fixed frame-interval in turn calculation. The most suitable number of fixed frame-interval depends on the correlation coefficients. Further mining of the spatial-transient patterns to separate the faulty areas and to enhance the detection sensitivity has been undertaken by PCA. The obtained experimental results have strongly validated the effectiveness and robustness of the proposed method by the abstract model and natural cracks on the impact damage area of the driven key. These quantitative analysis results have shown that the proposed method is a viable candidate as an alternative method to the Magnetic Particle Testing which is currently used in industry.

The proposed method has also shed new light not only on the deeper utilization of physical mechanisms of inductive heating propagation but also on better modeling and mining of the kinematic spatial-transient thermal patterns in quantitative defect detection. Future work will focus on the robustness and universality testing of the proposed method on more specimens and different thermal platforms.

Fig.16 The results of different signal processing method

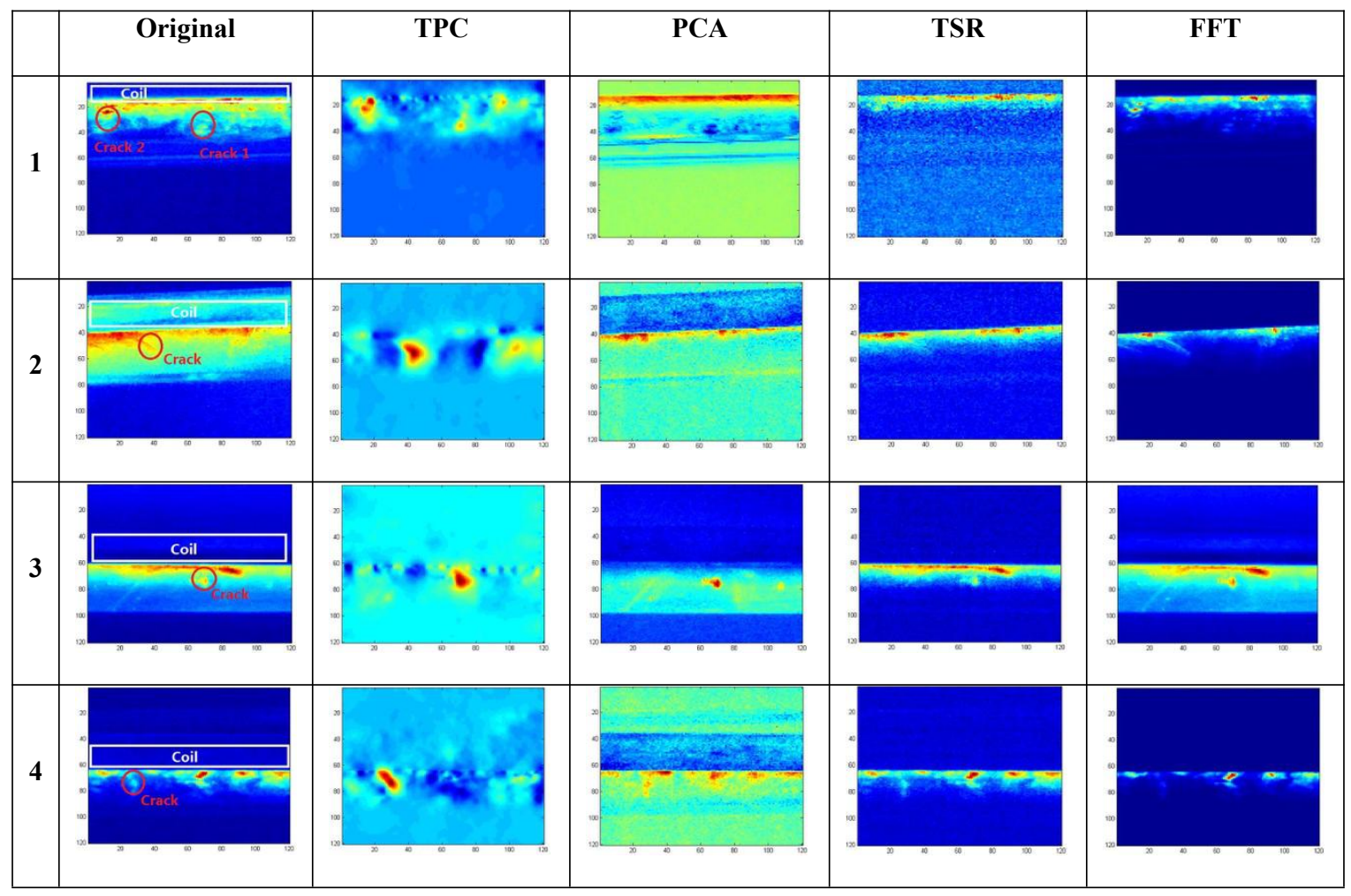




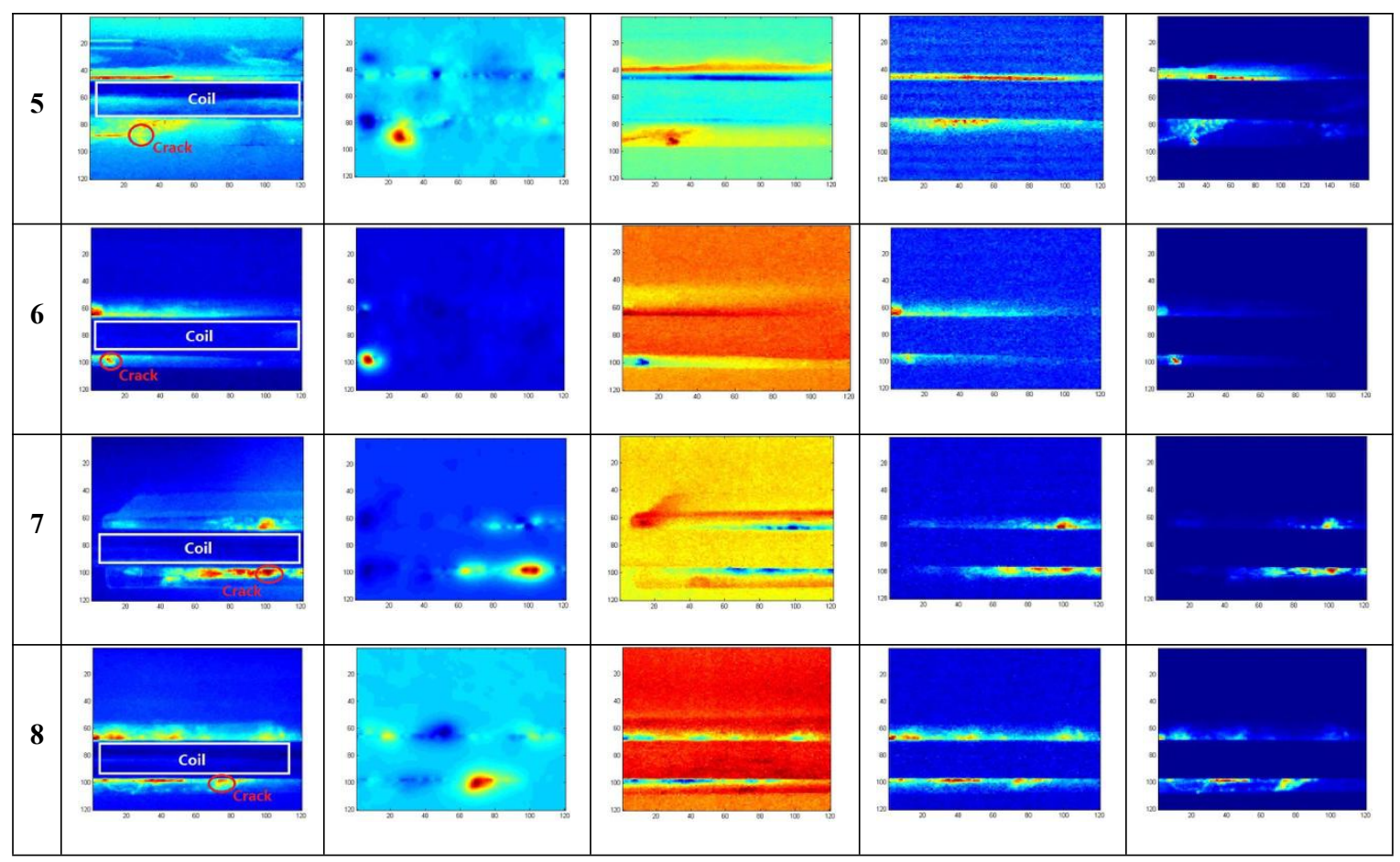

Table 2 The results of the quantitative analysis

\begin{tabular}{|l|l|l|l|l|l|l|l|l|l|}
\hline \multicolumn{2}{|c|}{} & $\mathbf{1}$ & $\mathbf{2}$ & $\mathbf{3}$ & $\mathbf{4}$ & $\mathbf{5}$ & $\mathbf{6}$ & $\mathbf{7}$ & $\mathbf{8}$ \\
\hline \multirow{2}{*}{ TPC } & F-SCORE(\%) & 85.7 & 80.0 & 100.0 & 100.0 & 100.0 & 100.0 & 80.0 & 100.0 \\
\cline { 2 - 10 } & SNR(dB) & 12.1 & 12.4 & 14.4 & 12.8 & 17.1 & 22.4 & 17.2 & 16.5 \\
\hline \multirow{2}{*}{ PCA } & F-SCORE(\%) & 0.0 & 0.0 & 100.0 & 0.0 & 33.3 & 100.0 & 0.0 & 0.0 \\
\cline { 2 - 10 } & SNR(dB) & $\mathbf{x}$ & $\mathbf{x}$ & 11.2 & $\mathbf{x}$ & 9.7 & 15.6 & $\mathbf{x}$ & $\mathbf{x}$ \\
\hline \multirow{2}{*}{ TSR } & F-SCORE(\%) & 0.0 & 0.0 & 33.3 & 28.6 & 0.0 & 66.7 & 40.0 & 33.3 \\
\cline { 2 - 10 } & SNR(dB) & $\mathbf{x}$ & $\mathbf{x}$ & 2.7 & -2.9 & $\mathbf{x}$ & 11.2 & 13.1 & 8.2 \\
\hline \multirow{2}{*}{ FFT } & F-SCORE(\%) & 50.0 & 66.7 & 50.0 & 0.0 & 40.0 & 100.0 & 40.0 & 33.3 \\
\cline { 2 - 10 } & SNR(dB) & 8.6 & -0.8 & 3.8 & $\mathbf{x}$ & 7.0 & 22.2 & 16.7 & 13.4 \\
\hline
\end{tabular}

\section{ACKNOWLEDGEMENT}

The work was supported by National Natural Science Foundation of China (No. 61401071, No. 61527803), Supported by NSAF (Grant No. U1430115) and EPSRC IAA Phase 2 funded project: "3D super-fast and portable eddy current pulsed thermography for railway inspection" (EP/K503885/1)

\section{REFERENCES}

[1] Vavilov V. Thermal NDT: historical milestones, state-of-the-art and trends[J]. Quantitative Infrared Thermography Journal, 2014, 11(1):66-83.

[2] Yang R, He Y. Optically and non-optically excited thermography for composites: A review[J]. Infrared Physics \& Technology, 2016, 75:26-50.

[3] Bagavathiappan S, Lahiri B B, Saravanan T, et al. Infrared thermography for condition monitoring - A review[J]. Infrared Physics \& Technology, 2013, 60(5):35-55.
[4] Bond L J. Through the looking glass: The future for NDE?[C]. 40th Annual Review of Progress in Quantitative Nondestructive Evaluation, 2014, vols: 33A \& 33B

[5] Zhang H, Sfarra S, Sarasini F, et al. Optical and Mechanical Excitation Thermography for Impact Response in Basalt-Carbon Hybrid Fiber-Reinforced Composite Laminates[J]. IEEE Transactions on Industrial Informatics, 2017, PP(99):1-1.

[6] DeVlieg G H. System and method for aircraft braking system usage monitoring: U.S. Patent 6,659,233[P]. 2003-12-9.

[7] O'Connell C T, Oyama H. Aircraft brake system: U.S. Patent 9,108,726[P]. 2015-8-18.

[8] Michael Kroes, James Rardon,"Aircraft Basic Science", seventh edition, Tata McGraw-Hill Education, 2010.

[9] ASTM Standard E192, Standard Reference Radiographs for Investment Steel Castings of Aerospace Applications, ASTM, West Conshohocken, 1999 [10] Sangid M D. The physics of fatigue crack initiation[J]. International journal of fatigue, 2013, 57: 58-72.

[11] Gao B, Woo W L, He Y, et al. Unsupervised Sparse Pattern Diagnostic of Defects with Inductive Thermography Imaging System[J]. IEEE Transactions on Industrial Informatics, 2015, 12(1):371-383.

[12] He Y, Yang R. Eddy Current Volume Heating Thermography and Phase Analysis for Imaging Characterization of Interface Delamination in CFRP[J]. IEEE Transactions on Industrial Informatics, 2015, 11(6):1287-1297. 
[13] Vavilov V P, Burleigh D D. Review of pulsed thermal NDT: Physical principles, theory and data processing $[\mathrm{J}]$. Ndt \& E International, 2015, 73(1):28-52.

[14] Gao B, He Y, Woo W L, et al. Multidimensional Tensor-Based Inductive Thermography with Multiple Physical Fields for Offshore Wind Turbine Gear Inspection[J]. IEEE Transactions on Industrial Electronics, 2016, 63(10):6305-6315.

[15] O. Lucia, P. Maussion, E. J. Dede, and J. M. Burdio, "Induction heating technology and its applications: past developments, current technology, and future challenges," IEEE Trans. Ind. Electron., vol. 61, no. 5, pp. 25092520, May 2014.

[16] O. Lucia, P. Maussion, E. J. Dede, and J. M. Burdio, "Introduction to the special section on induction heating systems," IEEE Trans. Ind. Electron.,

vol. 61 , no. 5, pp. 2504-2508, May 2014.

[17] Ibarra-Castanedo C, Tarpani J R, Maldague X P V. Nondestructive testing with thermography[J]. European Journal of Physics, 2013, 34(6): S91.

[18] Shepard, S.M., "Advances in pulse thermography", Thermosense XXIII SPIE Proceedings Vol. 4360, pp. 511-515, 2001.

[19] Gao B, Bai L, Woo W L, et al. Automatic defect identification of eddy current pulsed thermography using single channel blind source separation[J]. IEEE Transactions on Instrumentation and Measurement, 2014, 63(4): 913-922.

[20] Maldague X, Marinetti S. Pulse phase infrared thermography. J Appl Phys 1996;79(5):2694-8.

[21]Chatterjee K, Tuli S. Image enhancement in transient lock-in thermography through time series reconstruction and spatial slope correction. IEEE Trans Instrum Meas 2012;61(4):1079-89.

[22] Vrana J, Goldammer M, Baumann J, et al. Mechanisms and Models for Crack Detection with Induction Thermography[C]// American Institute of Physics, 2008:475-482.

[23] Dudzik S. Analysis of the accuracy of a neural algorithm for defect depth estimation using PCA processing from active thermography data[J]. Infrared Physics \& Technology, 2013, 56(36):1-7.

[24] Peng J, Tian G Y, Wang L, et al. Investigation into eddy current pulsed thermography for rolling contact fatigue detection and characterization[J]. Ndt \& E International, 2015, 74(6):72-80.

[25] Gao B, Li X, Woo W L, et al. Quantitative validation of Eddy current stimulated thermal features on surface crack[J]. Ndt \& E International, 2017, 85:1-12.

[26] Wang Y, Gao B, Tian G, et al. Diffusion and separation mechanism of transient electromagnetic and thermal fields[J]. International Journal of Thermal Sciences, 2016, 102: 308-318.

[27] Gao B, Woo W L, Tian G Y. Electromagnetic Thermography Nondestructive Evaluation: Physics-based Modeling and Pattern Mining[J]. Scientific reports, 2016, 6 .

[28] B. K. P. Horn and B. G. Schunck, "Determining optical flow," Artif. Intell., vol. 17, pp. 185-203, 1981.

[29] Jang G J, Lee T W. A maximum likelihood approach to single-channel source separation[J]. Journal of Machine Learning Research, 2003, 4(Dec): 1365-1392.

[30] Lee Rodgers J, Nicewander W A. Thirteen ways to look at the correlation coefficient[J]. The American Statistician, 1988, 42(1): 59-66.

[31] Kleijnen J P C. Design and Analysis of Monte Carlo Experiments[J]. Social Science Electronic Publishing, 2004, 2004-17(5):497-516.

[32] He Y, Pan M, Tian G Y, et al. Eddy current pulsed phase thermography for subsurface defect quantitatively evaluation[J]. Applied Physics Letters, 2013, 103(14):054103-2120.

[33] Powers D M. Evaluation: from precision, recall and F-measure to ROC, informedness, markedness and correlation[J]. Journal of Machine Learning Technologies. 2011,2(1): 37-63.

[34] Omar M A, Zhou Y. A quantitative review of three flash thermography processing routines[J]. Infrared Physics \& Technology, 2008, 51(4): 300-306.

[35] Krapivsky P L, Redner S, Ben-Naim E. A Kinetic View of Statistical Physics[M]// A kinetic view of statistical physics /. Cambridge University Press, 2010:831-832.

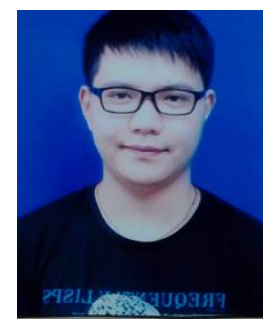

Yizhe Wang received his B.Sc.degree in physics from Shenyang Normal University, Shenyang, China (2008-2012), and the M.E. degree in instrument science and technology from University of Electronic Science and Technology of China (UESTC), Chengdu, China (2012-2014). He is currently working toward the Ph.D. degree in quantitative fatigue assessment and health state monitoring using infrared thermography at UESTC, Chengdu, China and Laval University, Quebec, Canada. His current research interests include sensor design, fatigue damage, quantitative non-destructive testing and evaluation, sensor signal processing and machine learning.

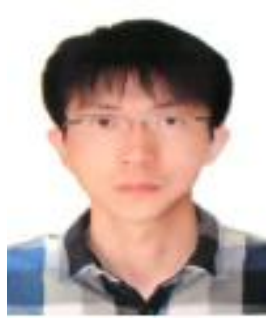

Bin Gao (M' 12-SM' 14) received his B.Sc. degree in communications and signal processing from Southwest Jiao Tong University (2001-2005), China, MSc degree in communications and signal processing with Distinction and $\mathrm{PhD}$ degree from Newcastle University, UK (2006-2011). He worked as a Research Associate (2011-2013) with the same university on wearable acoustic sensor technology. Currently, he is a Professor with the School of Automation Engineering, University of Electronic Science and Technology of China (UESTC), China. His research interests include sensor signal processing, machine learning, social signal processing, nondestructive testing and evaluation where he actively publishes in these areas. $\mathrm{He}$ has published over 60 papers on these topics on various journals and international conference proceedings. He has coordinated several research projects from National Natural Science Foundation of China.

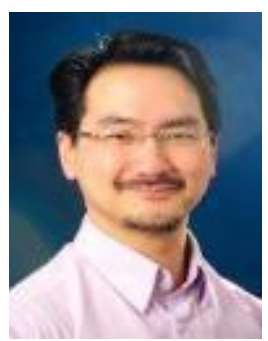

Wai Lok Woo was born in Malaysia. He received the BEng degree (1st Class Hons.) in Electrical and Electronics Engineering and the $\mathrm{PhD}$ degree from the Newcastle University, UK. He was awarded the IEE Prize and the British Scholarship to continue his research work. He is currently a Senior Lecturer and Director of Operations with the School of Electrical and Electronic Engineering. His major research is in the mathematical theory and algorithms for nonlinear signal and image processing. This includes areas of machine learning for signal processing, blind source separation, multidimensional signal processing, signal/image deconvolution and restoration. He has an extensive portfolio of relevant research supported by a variety of funding agencies. He has published over 250 papers on these topics on various journals and international conference proceedings. Currently, he is Associate Editor of several international journals and has served as lead-editor of journals' special issues.

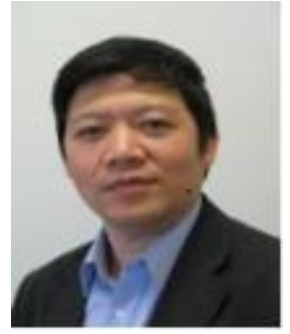

Gui Yun Tian (M'01-SM'03) received the B.Sc. degree in metrology and instrumentation and M.Sc. degree in precision engineering from the University of Sichuan, Chengdu, China, in 1985 and 1988, respectively, and the $\mathrm{Ph} . \mathrm{D}$. degree from the University of Derby, Derby, U.K., in 1998. From 2000 to 2006, he was a Lecturer, Senior Lecturer, Reader, Professor, and Head of the group of Systems Engineering, respectively, with the University of Huddersfield, U.K. Since 2007, he has been based at Newcastle University, Newcastle upon Tyne, U.K., where he has been Chair Professor in Sensor Technologies. Currently, He is also an adjunct professor with School of Automation Engineering, University of Electronic Science and Technology of China. He has coordinated several research projects from the Engineering and Physical Sciences Research Council (EPSRC), Royal Academy of Engineering and FP7, on top of this he also has good collaboration with leading industrial companies such as Airbus, Rolls Royce, BP, nPower, Networkrail and TWI. 


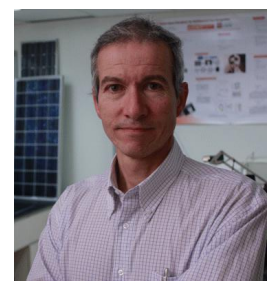

Xavier Maldague is a full professor in the Department of Electrical and Computing Engineering, university Laval, Québec City, Canada (since 1989, head of the Dept. 2003-2008). He has trained over 50 graduate students (M.Sc. and Ph.D.) and has more than 300 publications. His research interests are infrared thermography, NonDestructive Evaluation (NDE) techniques and vision / digital systems for industrial inspection. He holds a Tier 1 Canada Research Chair in infrared vision. He chairs the Quantitative Infrared Thermography (QIRT) Council. $\mathrm{He}$ is a fellow of the Canada Engineering Institute, an Honorary Fellow of the Indian Society of Nondestructive Testing and a fellow of the Alexander von Humbolt Foundation in Germany. Within IEEE Canada, he was Co- Editor of the IEEE Canadian Journal of Electrical and Computer Engineering (2003-2007). In 2002-2004 and in 2015-2017, he chaired the IEEE Quebec City Section. In 2004, he was awarded the IEEE J. J. Archambault Award for his dedication to IEEE and his contributions to engineering progress. In 2008-2011, he was the IEEE Canada Publications and Communications Group Chair.

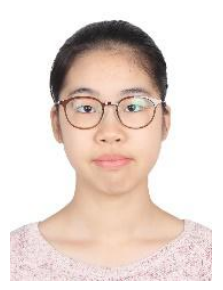

Li Zheng is currently pursuing the B.Sc. degree in the UESTC UoG Joint school, University of Electronic Science and Technology of China, Chengdu, China. Her research interests include nondestructive testing and image processing.

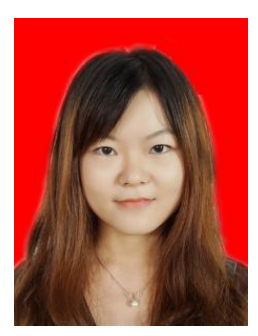

Zheyou Guo is the third year student in the University of Electronic Science and Technology of China. She is pursuing the B.Sc. degree in Electrical\&information Engineering at the UESTC and Uog (University of Glasgow) Joint school, Chengdu, China. Her research interests include nondestructive testing and deep learning.

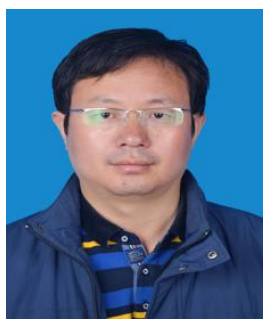

Yuyu Zhu received the B.S. degree in automation from the Southwest University of Science and Technology, China, in 2002, and the M.Sc. degree in control theory and control engineering with the Southwest University of Science and Technology, in 2009. He is currently pursuing the Ph.D. degree with the University of Electronic Science and Technology of China. He is an Associate Professor with the School on Monitoring and Control Technology and Power Electronics Technology. His research interests include nondestructive testing and evaluation, power electronics technology. 